\title{
APPLICATIONS OF OSTROWSKI'S VERSION OF THE GRÜSS INEQUALITY FOR TRAPEZOID TYPE RULES
}

\author{
N. S. BARNETT AND S. S. DRAGOMIR
}

Abstract. Some applications of the Ostrowski inequality and a perturbed version of it for integral inequalities of the trapezoid type are given.

\section{Introduction}

In [1], A. Ostrowski proved the following inequality of the Grüss type,

$$
\begin{aligned}
& \left|\frac{1}{b-a} \int_{a}^{b} f(x) g(x) d x-\frac{1}{b-a} \int_{a}^{b} f(x) d x \cdot \frac{1}{b-a} \int_{a}^{b} g(x) d x\right| \\
\leq & \frac{1}{8}(b-a)(M-m)\left\|f^{\prime}\right\|_{[a, b], \infty}
\end{aligned}
$$

provided $g$ is integrable on $[a, b]$ and satisfies the condition,

$$
-\infty<m \leq g(x) \leq M<\infty \text { for a.e. } x \in[a, b]
$$

and $f$ is absolutely continuous on $[a, b]$ with $f^{\prime} \in L_{\infty}[a, b]$.

The constant $\frac{1}{8}$ is the best possible in (1.1) in the sense that it cannot be replaced by a smaller one.

In this paper we present some applications of (1.1) as well as a perturbed version of it that can also be applied to create some useful integral inequalities.

\section{Integral Inequalities}

The following trapezoid type result for $n$-time differentiable functions has been obtained in [2].

Received December 11, 2002; revised July 20, 2005.

2000 Mathematics Subject Classification. Primary 26D15; Secondary 41A55.

Key words and phrases. Ostrowski's inequality, Grüss type inequalities, trapezoid type inequalities. 
Lemma 1. Let $f:[a, b] \rightarrow \mathbb{R}$ be a mapping such that the derivative $f^{(n-1)}(n \geq 1)$ is absolutely continuous on $[a, b]$, then for any $x \in[a, b]$,

$$
\begin{aligned}
\int_{a}^{b} f(t) d t= & \sum_{k=0}^{n-1} \frac{1}{(k+1) !}\left[(x-a)^{k+1} f^{(k)}(a)+(-1)^{k}(b-x)^{k+1} f^{(k)}(b)\right] \\
& +\frac{1}{n !} \int_{a}^{b}(x-t)^{n} f^{(n)}(t) d t
\end{aligned}
$$

Some useful particular cases follow.

(1) For $n=1$, we can retrieve the identity (see for example [2]),

$$
\int_{a}^{b} f(t) d t=(x-a) f(a)+(b-x) f(b)+\int_{a}^{b}(x-t) f^{\prime}(t) d t
$$

for each $x \in[a, b]$.

(2) For $n=2$, we have (see for example [2]),

$$
\begin{aligned}
\int_{a}^{b} f(t) d t= & (x-a) f(a)+(b-x) f(b) \\
& +\frac{1}{2}\left[(x-a)^{2} f^{\prime}(a)+(b-x)^{2} f^{\prime}(b)\right]+\frac{1}{2} \int_{a}^{b}(x-t)^{2} f^{\prime \prime}(t) d t
\end{aligned}
$$

If in $(2.2)$ we choose $x=\frac{a+b}{2}$, then we get the trapezoid identity,

$$
\int_{a}^{b} f(t) d t=\frac{f(b)+f(a)}{2}(b-a)+\int_{a}^{b}\left(\frac{a+b}{2}-t\right) f^{\prime}(t) d t
$$

while the same choice of $x$ will produce, in (2.3), the following perturbed version of the trapezoid identity,

$$
\begin{aligned}
\int_{a}^{b} f(t) d t= & \frac{f(b)+f(a)}{2}(b-a)+\frac{(b-a)^{2}}{8}\left[f^{\prime}(a)-f^{\prime}(b)\right] \\
& +\frac{1}{2} \int_{a}^{b}\left(t-\frac{a+b}{2}\right)^{2} f^{\prime \prime}(t) d t
\end{aligned}
$$

Consider now the following results.

Theorem 1. Let $f:[a, b] \rightarrow \mathbb{R}$ be a mapping such that the derivative $f^{(n-1)}(n \geq 1)$ is absolutely continuous on $[a, b]$ and there exist real numbers $\gamma, \Gamma$ such that $-\infty<\gamma \leq$ 
$f^{(n)}(x) \leq \Gamma<\infty$ for a.e. $x \in[a, b]$, then,

$$
\begin{aligned}
& \mid \int_{a}^{b} f(t) d t-\sum_{k=0}^{n-1} \frac{1}{(k+1) !}\left[(x-a)^{k+1} f^{(k)}(a)+(-1)^{k}(b-x)^{k+1} f^{(k)}(b)\right] \\
& \quad-\frac{(x-a)^{n+1}+(-1)^{n}(b-x)^{n+1}}{(n+1) !}\left[f^{(n-1)} ; a, b\right] \mid \\
& \leq \frac{1}{8(n-1) !}(b-a)^{2}(\Gamma-\gamma)\left[\frac{1}{2}(b-a)+\left|x-\frac{a+b}{2}\right|\right]^{n-1}
\end{aligned}
$$

for any $x \in[a, b]$, where $[h ; a, b]=\frac{h(b)-h(a)}{b-a}$ is the divided difference of $h$ on $[a, b]$.

Proof. If we use Ostrowski's inequality (1.1) we may write,

$$
\begin{aligned}
& \left|\frac{1}{b-a} \int_{a}^{b}(x-t)^{n} f^{(n)}(t) d t-\frac{1}{b-a} \int_{a}^{b}(x-t)^{n} d t \cdot \frac{1}{b-a} \int_{a}^{b} f^{(n)}(t) d t\right| \\
\leq & \frac{1}{8}(b-a)(\Gamma-\gamma) n \sup _{t \in[a, b]}|x-t|^{n-1} \\
= & \frac{n}{8}(b-a)(\Gamma-\gamma)[\max (x-a, b-x)]^{n-1} \\
= & \frac{n}{8}(b-a)(\Gamma-\gamma)\left[\frac{1}{2}(b-a)+\left|x-\frac{a+b}{2}\right|\right]^{n-1}
\end{aligned}
$$

giving

$$
\begin{aligned}
& \left|\int_{a}^{b}(x-t)^{n} f^{(n)}(t) d t-\frac{(x-a)^{n+1}+(-1)^{n}(b-x)^{n+1}}{n+1}\left[f^{(n-1)} ; a, b\right]\right| \\
\leq & \frac{n}{8}(b-a)^{2}(\Gamma-\gamma)\left[\frac{1}{2}(b-a)+\left|x-\frac{a+b}{2}\right|\right]^{n-1} .
\end{aligned}
$$

If we divide this by $n$ ! and use the representation (2.1), we obtain the desired inequality $(2.6)$.

Remark 1. If $n=1$ in (2.6), we deduce,

$$
\begin{aligned}
& \left|\int_{a}^{b} f(t) d t-(x-a) f(a)-(b-x) f(b)-(b-a)\left(x-\frac{a+b}{2}\right)[f ; a, b]\right| \\
\leq & \frac{1}{8}(b-a)^{2}(\Gamma-\gamma),
\end{aligned}
$$

which implies the trapezoid inequality,

$$
\left|\int_{a}^{b} f(t) d t-\frac{f(b)+f(a)}{2}(b-a)\right| \leq \frac{1}{8}(b-a)^{2}(\Gamma-\gamma) .
$$


It has been shown in various papers that $\frac{1}{8}$ is a sharp constant (see [4], and [3]).

Remark 2. Further work has yet to be done on comparing, for any $x \in[a, b]$, the bounds provided by (2.6) and the bound,

$$
\frac{\Gamma-\gamma}{2} \cdot \frac{1}{n !} I(x, n),
$$

where

$$
\begin{array}{r}
I(x, n):=\frac{1}{(n+1) \sqrt{2 n+1}}\left\{n^{2}(b-a)\left[(x-a)^{2 n+1}+(b-x)^{2 n+1}\right]\right. \\
\left.+(2 n+1)(x-a)(b-x)\left[(x-a)^{n}+(b-x)^{n}\right]^{2}\right\}^{\frac{1}{2}}
\end{array}
$$

has been obtained in [2].

The Ostrowski inequality (1.1) can also be applied in the following way.

Theorem 2. Let $f:[a, b] \rightarrow \mathbb{R}$ be a mapping such that the derivative $f^{(n)}$ is absolutely continuous and $f^{(n+1)} \in L_{\infty}[a, b]$, then,

$$
\begin{aligned}
& \mid \int_{a}^{b} f(t) d t-\sum_{k=0}^{n-1} \frac{1}{(k+1) !}\left[(x-a)^{k+1} f^{(k)}(a)+(-1)^{k}(b-x)^{k+1} f^{(k)}(b)\right] \\
& -\frac{(x-a)^{n+1}+(-1)^{n}(b-x)^{n+1}}{(n+1) !}\left[f^{(n-1)} ; a, b\right] \mid \\
& \leq \begin{cases}\frac{1}{8 n !}(b-a)^{2}\left[\frac{1}{2}(b-a)+\left|x-\frac{a+b}{2}\right|\right]^{n}\left\|f^{(n+1)}\right\|_{[a, b], \infty} \text { if } n \text { is even, } \\
\frac{1}{8 n !}(b-a)^{2}\left[(x-a)^{n}+(b-x)^{n}\right]\left\|f^{(n+1)}\right\|_{[a, b], \infty} & \text { if } n \text { is odd. }\end{cases}
\end{aligned}
$$

Proof. For $n=2 k$, consider $h_{2 k}(t)=(x-t)^{2 k}$. It is obvious that

$$
\begin{gathered}
\inf _{t \in[a, b]} h_{2 k}(t)=0, \\
\sup _{t \in[a, b]} h_{2 k}(t)=\max \left[(x-a)^{2 k},(b-x)^{2 k}\right]=[\max (x-a, b-x)]^{2 k} \\
=\left[\frac{1}{2}(b-a)+\left|x-\frac{a+b}{2}\right|\right]^{2 k} .
\end{gathered}
$$


If we now apply Ostrowski's inequality (1.1) for $h_{2 k}$ and $f^{(2 k)}$, we deduce

$$
\begin{aligned}
& \left|\frac{1}{b-a} \int_{a}^{b}(x-t)^{2 k} f^{(2 k)}(t) d t-\frac{1}{b-a} \int_{a}^{b}(x-t)^{2 k} d t \cdot \frac{1}{b-a} \int_{a}^{b} f^{(2 k)}(t) d t\right| \\
\leq & \frac{1}{8}(b-a)\left[\frac{1}{2}(b-a)+\left|x-\frac{a+b}{2}\right|\right]^{2 k}\left\|f^{(2 k+1)}\right\| \|_{[a, b], \infty},
\end{aligned}
$$

by a similar argument to that in Theorem 1, proving the first part of (2.9).

For $n=2 k+1$, consider $h_{2 k+1}(t)=(x-t)^{2 k+1}$, then

$$
h_{2 k+1}^{\prime}(t)=-(2 k+1)(x-t)^{2 k}
$$

showing that $h_{2 k+1}$ is decreasing on $[a, b]$, and thus,

$$
\inf _{t \in[a, b]} h_{2 k+1}(t)=h_{2 k+1}(b)=(x-b)^{2 k+1}=-(b-x)^{2 k+1}
$$

and

$$
\sup _{t \in[a, b]} h_{2 k+1}(t)=h_{2 k+1}(a)=(x-a)^{2 k+1} .
$$

Now apply Ostrowski's inequality (1.1) for $h_{2 k+1}$ and $f^{(2 k+1)}$ we get,

$$
\begin{aligned}
& \mid \frac{1}{b-a} \int_{a}^{b}(x-t)^{2 k+1} f^{(2 k+1)}(t) d t \\
& -\frac{1}{b-a} \int_{a}^{b}(x-t)^{2 k+1} d t \cdot \frac{1}{b-a} \int_{a}^{b} f^{(2 k+1)}(t) d t \mid \\
\leq & \frac{1}{8}(b-a)\left[(x-a)^{2 k+1}+(b-x)^{2 k+1}\right]\left\|f^{(2 k+1)}\right\|_{[a, b], \infty},
\end{aligned}
$$

giving, by a similar procedure to that in Theorem 1, the second part of (2.9).

\section{A Perturbed Version}

The following result holds.

Theorem 3. Let $f:[a, b] \rightarrow \mathbb{R}$ be an absolutely continuous function on $[a, b]$ such that the derivative $f^{\prime}:[a, b] \rightarrow \mathbb{R}$ satisfies the condition:

$$
-\infty<\gamma \leq f^{\prime}(x) \leq \Gamma<\infty \text { for a.e. } x \in[a, b] .
$$

If $g:[a, b] \rightarrow \mathbb{R}$ is such that,

$$
-\infty<m \leq g(x) \leq M<\infty \text { for a.e. } x \in[a, b]
$$


then,

$$
\begin{aligned}
& \mid \frac{1}{b-a} \int_{a}^{b} f(x) g(x) d x-\frac{1}{b-a} \int_{a}^{b} f(x) d x \cdot \frac{1}{b-a} \int_{a}^{b} g(x) d x \\
& -\frac{\gamma+\Gamma}{2} \cdot \frac{1}{b-a} \int_{a}^{b}\left(x-\frac{a+b}{2}\right) g(x) d x \mid \leq \frac{1}{16}(b-a)(M-m)(\Gamma-\gamma) .
\end{aligned}
$$

The constant $\frac{1}{16}$ is the best possible.

Proof. We know that,

$$
\begin{aligned}
& \quad\left|\frac{1}{b-a} \int_{a}^{b} h(x) g(x) d x-\frac{1}{b-a} \int_{a}^{b} h(x) d x \cdot \frac{1}{b-a} \int_{a}^{b} g(x) d x\right| \\
& \leq \frac{1}{8}(b-a)(M-m)\left\|h^{\prime}\right\|_{[a, b], \infty},
\end{aligned}
$$

provided $-\infty<m \leq g(x) \leq M<\infty$ for a.e. $x \in[a, b]$.

Choose $h(x)=f(x)-\frac{\gamma+\Gamma}{2}(x-\alpha), \alpha \in \mathbb{R}$, then,

$$
h^{\prime}(x)=f^{\prime}(x)-\frac{\gamma+\Gamma}{2}
$$

and since

$$
\left|h^{\prime}(x)\right| \leq \frac{\Gamma-\gamma}{2}
$$

for a.e. $x \in[a, b]$, we have,

$$
\begin{aligned}
& \mid \frac{1}{b-a} \int_{a}^{b}\left[f(x)-\frac{\gamma+\Gamma}{2}(x-\alpha)\right] g(x) d x \\
& -\frac{1}{b-a} \int_{a}^{b}\left[f(x)-\frac{\gamma+\Gamma}{2}(x-\alpha)\right] d x \cdot \frac{1}{b-a} \int_{a}^{b} g(x) d x \mid \\
& \leq \frac{1}{16}(b-a)(M-m)(\Gamma-\gamma) .
\end{aligned}
$$

However,

$$
\begin{aligned}
& \frac{1}{b-a} \int_{a}^{b}\left[f(x)-\frac{\gamma+\Gamma}{2}(x-\alpha)\right] g(x) d x \\
= & \frac{1}{b-a} \int_{a}^{b} f(x) g(x) d x-\frac{\gamma+\Gamma}{2} \cdot \frac{1}{b-a} \int_{a}^{b}(x-\alpha) g(x) d x
\end{aligned}
$$


and

$$
\begin{aligned}
& \frac{1}{b-a} \int_{a}^{b}\left[f(x)-\frac{\gamma+\Gamma}{2}(x-\alpha)\right] d x \cdot \frac{1}{b-a} \int_{a}^{b} g(x) d x \\
= & \frac{1}{b-a} \int_{a}^{b} f(x) d x \cdot \frac{1}{b-a} \int_{a}^{b} g(x) d x \\
& -\frac{\gamma+\Gamma}{2} \cdot \frac{1}{b-a} \int_{a}^{b}(x-\alpha) d x \cdot \frac{1}{b-a} \int_{a}^{b} g(x) d x .
\end{aligned}
$$

By (3.5) we deduce,

$$
\begin{aligned}
& \mid \frac{1}{b-a} \int_{a}^{b} f(x) g(x) d x-\frac{\gamma+\Gamma}{2} \cdot \frac{1}{b-a} \int_{a}^{b}(x-\alpha) g(x) d x \\
& -\frac{1}{b-a} \int_{a}^{b} f(x) d x \cdot \frac{1}{b-a} \int_{a}^{b} g(x) d x+\frac{\gamma+\Gamma}{2} \frac{1}{b-a} \int_{a}^{b}(x-\alpha) d x \cdot \frac{1}{b-a} \int_{a}^{b} g(x) d x \mid \\
& \leq \frac{1}{16}(b-a)(M-m)(\Gamma-\gamma) .
\end{aligned}
$$

Now, observe that,

$$
\begin{aligned}
& \frac{\gamma+\Gamma}{2} \cdot \frac{1}{b-a} \int_{a}^{b}(x-\alpha) g(x) d x \\
& -\frac{\gamma+\Gamma}{2} \cdot \frac{1}{b-a} \int_{a}^{b}(x-\alpha) d x \cdot \frac{1}{b-a} \int_{a}^{b} g(x) d x \\
= & \frac{\gamma+\Gamma}{2} \cdot \frac{1}{(b-a)^{2}}\left\{( b - a ) \left[\int_{a}^{b} x g(x) d x-\alpha \int_{a}^{b} g(x) d x\right.\right. \\
& \left.\left.\quad-\left[\int_{a}^{b} x d x-\alpha(b-a)\right] \int_{a}^{b} g(x) d x\right]\right\} \\
= & \frac{\gamma+\Gamma}{2} \cdot \frac{1}{(b-a)^{2}}\left[(b-a) \int_{a}^{b} x g(x) d x-\int_{a}^{b} x d x \cdot \int_{a}^{b} g(x) d x\right] \\
= & \frac{\gamma+\Gamma}{2}\left[\frac{1}{b-a} \int_{a}^{b} x g(x) d x-\frac{a+b}{2} \cdot \frac{1}{b-a} \int_{a}^{b} g(x) d x\right]
\end{aligned}
$$

and by (3.6) we deduce the desired result.

The fact that $\frac{1}{16}$ is the best constant, follows by Ostrowski's result on choosing $\gamma=$ - $\left\|f^{\prime}\right\|_{[a, b], \infty}, \Gamma=\left\|f^{\prime}\right\|_{[a, b], \infty}$. We omit the details.

In what follows, an application of the above perturbed version of Ostrowski's inequality is given. 
Using Lemma 1, we have the identity, (see also [2]),

$$
\begin{aligned}
\int_{a}^{b} f(t) d t= & \sum_{k=0}^{n-1} \frac{1}{(k+1) ! 2^{k+1}}(b-a)^{k+1}\left[f^{(k)}(a)+(-1)^{k} f^{(k)}(b)\right] \\
& +\frac{1}{n !} \int_{a}^{b}\left(\frac{a+b}{2}-t\right)^{n} f^{(n)}(t) d t
\end{aligned}
$$

We can further state the following result.

Theorem 4. Let $f:[a, b] \rightarrow \mathbb{R}$ be a mapping such that the derivative $f^{(n-1)} \quad(n \geq 1)$ is absolutely continuous on $[a, b]$ and there exists the real numbers $m, M$ such that $-\infty<$ $m \leq f^{(n)}(x) \leq M<\infty$ for a.e., $x \in[a, b]$. We then have the inequality:

$$
\begin{aligned}
& \left|\int_{a}^{b} f(t) d t-\sum_{k=0}^{n-1} \frac{(b-a)^{k+1}}{(k+1) ! 2^{k+1}}\left[f^{(k)}(a)+(-1)^{k} f^{(k)}(b)\right]-\frac{(b-a)^{n+1}}{2^{n}(n+1) !}\left[f^{(n-1)} ; b, a\right]\right| \\
\leq & \frac{(b-a)^{n+1}}{(n-1) ! 2^{n+2}}(M-m)
\end{aligned}
$$

if $n$ is even and

$$
\begin{aligned}
& \mid \int_{a}^{b} f(t) d t-\sum_{k=0}^{n-1} \frac{(b-a)^{k+1}}{(k+1) ! 2^{k+1}}\left[f^{(k)}(a)+(-1)^{k} f^{(k)}(b)\right] \\
& \quad+\frac{(b-a)^{n}}{(n-1) ! 2^{n-1}}\left[\left[f^{(n-2)} ; b, a\right]-\frac{f^{(n-1)}(b)+f^{(n-1)}(a)}{2}\right] \mid \\
& \leq \frac{(b-a)^{n+1}}{(n-1) ! 2^{n+3}}(M-m)
\end{aligned}
$$

if $n$ is odd.

Proof. The proof is by application of Theorem 3 for $g \rightarrow f^{(n)}$ and $f \rightarrow\left(\frac{a+b}{2}-\cdot\right)^{n}$. We first observe that,

$$
\begin{gathered}
\int_{a}^{b}\left(\frac{a+b}{2}-t\right)^{n} d t=\frac{(b-a)^{n+1}\left[1+(-1)^{n}\right]}{2^{n+1}(n+1)}, \\
\frac{1}{b-a} \int_{a}^{b} f^{(n)}(t) d t=\left[f^{(n-1)} ; b, a\right]
\end{gathered}
$$


OSTROWSKI'S VERSION OF THE GRÜSS INEQUALITY

171

$$
\begin{aligned}
& \gamma=\inf _{t \in[a, b]}\left[\frac{d}{d t}\left(\frac{a+b}{2}-t\right)^{n}\right]=\left\{\begin{array}{l}
-n\left(\frac{b-a}{2}\right)^{n-1} \text { if } n \text { is even, } \\
-n\left(\frac{b-a}{2}\right)^{n-1} \text { if } n \text { is odd, }
\end{array}\right. \\
& \Gamma=\sup _{t \in[a, b]}\left[\frac{d}{d t}\left(\frac{a+b}{2}-t\right)^{n}\right]= \begin{cases}n\left(\frac{b-a}{2}\right)^{n-1} & \text { if } n \text { is even, } \\
0 & \text { if } n \text { is odd },\end{cases}
\end{aligned}
$$

and then,

$$
\frac{\gamma+\Gamma}{2}= \begin{cases}0 & \text { if } n \text { is even } \\ -n\left(\frac{b-a}{2}\right)^{n-1} & \text { if } n \text { is odd }\end{cases}
$$

and

$$
\Gamma-\gamma=\left\{\begin{array}{l}
2 n\left(\frac{b-a}{2}\right)^{n-1} \text { if } n \text { is even, } \\
n\left(\frac{b-a}{2}\right)^{n-1} \text { if } n \text { is odd. }
\end{array}\right.
$$

Also,

$$
\begin{aligned}
& \int_{a}^{b}\left(\frac{a+b}{2}-t\right) f^{(n)}(t) d t \\
& =\left.f^{(n-1)}(t)\left(\frac{a+b}{2}-t\right)\right|_{a} ^{b}+\int_{a}^{b} f^{(n-1)}(t) d t \\
& =-(b-a) \frac{f^{(n-1)}(b)+f^{(n-1)}(a)}{2}+\left[f^{(n-2)} ; b, a\right](b-a) .
\end{aligned}
$$

Now, when $n$ is even, we have,

$$
\begin{aligned}
& \left|\int_{a}^{b}\left(\frac{a+b}{2}-t\right)^{n} f^{(n)}(t) d t-\int_{a}^{b}\left(\frac{a+b}{2}-t\right)^{n} d t \cdot \frac{1}{b-a} \int_{a}^{b} f^{(n)}(t) d t\right| \\
\leq & \frac{1}{16}(b-a)^{2}\left[2 n\left(\frac{b-a}{2}\right)^{n-1}\right](M-m),
\end{aligned}
$$

i.e.,

$$
\left|\int_{a}^{b}\left(\frac{a+b}{2}-t\right)^{n} f^{(n)}(t) d t-\frac{(b-a)^{n+1}}{2^{n}(n+1) !} \cdot\left[f^{(n-1)} ; b, a\right]\right| \leq \frac{n(b-a)^{n+1}(M-m)}{2^{n+2}} .
$$


Dividing by $n$ ! and using (3.7), we get (3.8).

Now, when $n$ is odd, we have,

$$
\begin{aligned}
& \mid \int_{a}^{b}\left(\frac{a+b}{2}-t\right)^{n} f^{(n)}(t) d t-\int_{a}^{b}\left(\frac{a+b}{2}-t\right)^{n} d t \cdot \frac{1}{b-a} \int_{a}^{b} f^{(n)}(t) d t \\
& \quad+n\left(\frac{b-a}{2}\right)^{n-1}\left[(b-a)\left[f^{(n-2)} ; b, a\right]-(b-a) \frac{f^{(n-1)}(b)+f^{(n-1)}(a)}{2}\right] \mid \\
& \leq \frac{1}{16}(b-a)^{2}(M-m) n\left(\frac{b-a}{2}\right)^{n-1},
\end{aligned}
$$

i.e.,

$$
\begin{aligned}
& \quad\left|\int_{a}^{b}\left(\frac{a+b}{2}-t\right)^{n} f^{(n)}(t) d t+\frac{n(b-a)^{n}}{2^{n-1}}\left[\left[f^{(n-2)} ; b, a\right]-\frac{f^{(n-1)}(b)+f^{(n-1)}(a)}{2}\right]\right| \\
& \leq \frac{n(b-a)^{n+1}(M-m)}{2^{n+3}},
\end{aligned}
$$

and dividing by $n$ ! and using (3.7) we have (3.9).

The theorem is thus proved.

The second approach is incorporated in the following theorem.

Theorem 5. Let $f:[a, b] \rightarrow \mathbb{R}$ be a mapping such that the derivative $f^{(n)}$ is absolutely continuous on $[a, b]$ and assume that there exist constants $\phi, \Phi \in \mathbb{R}$ such that $-\infty<\phi \leq$ $f^{(n+1)}(x) \leq \Phi<\infty$ for a.e. $x \in[a, b]$, then,

$$
\begin{aligned}
& \mid \int_{a}^{b} f(t) d t-\sum_{k=0}^{n-1} \frac{(b-a)^{k+1}}{(k+1) ! 2^{k+1}}\left[f^{(k)}(a)+(-1)^{k} f^{(k)}(b)\right] \\
& -\frac{\left[1+(-1)^{n}\right](b-a)^{n+1}}{2^{n+1}(n+1) !}\left[f^{(n-1)} ; b, a\right]+\frac{\phi+\Phi}{2} \cdot \frac{\left[(-1)^{n}+1\right](b-a)^{n+2}}{2^{n+2}(n+2) n !} \mid \\
& \leq\left\{\begin{array}{l}
\frac{(b-a)^{n+2}}{2^{n+4} n !}(\Phi-\phi) \text { if } n \text { is even, } \\
\frac{(b-a)^{n+2}}{2^{n+3} n !}(\Phi-\phi) \text { if } n \text { is odd. }
\end{array}\right.
\end{aligned}
$$

Proof. We apply Theorem 3 for the choices $g \rightarrow\left(\frac{a+b}{2}-\cdot\right)^{n}$ and $f \rightarrow f^{(n)}$. We observe that,

$$
\begin{aligned}
\int_{a}^{b}\left(t-\frac{a+b}{2}\right)\left(\frac{a+b}{2}-t\right)^{n} d t & =-\int_{a}^{b}\left(\frac{a+b}{2}-t\right)^{n+1} d t \\
& =-\frac{(b-a)^{n+2}\left[(-1)^{n+1}+1\right]}{2^{n+2}(n+2)} .
\end{aligned}
$$


By (3.3) we then get,

$$
\begin{aligned}
& \mid \int_{a}^{b}\left(\frac{a+b}{2}-t\right)^{n} f^{(n)}(t) d t-\int_{a}^{b}\left(\frac{a+b}{2}-t\right)^{n} d t \cdot \frac{1}{b-a} \int_{a}^{b} f^{(n)}(t) d t \\
& -\frac{\phi+\Phi}{2} \int_{a}^{b}\left(t-\frac{a+b}{2}\right)\left(\frac{a+b}{2}-t\right)^{n} d t \mid \\
& \leq \frac{1}{16}(b-a)^{2}(\Phi-\phi)\left\{\begin{array}{l}
\frac{(b-a)^{n}}{2^{n}} \text { if } n \text { is even, } \\
\frac{(b-a)^{n}}{2^{n-1}} \text { if } n \text { is odd, }
\end{array}\right.
\end{aligned}
$$

that is,

$$
\begin{aligned}
& \mid \int_{a}^{b}\left(\frac{a+b}{2}-t\right)^{n} f^{(n)}(t) d t-\frac{(b-a)^{n+1}\left[1+(-1)^{n}\right]}{2^{n+1}(n+1)}\left[f^{(n-1)} ; b, a\right] \\
& +\frac{\phi+\Phi}{2} \cdot \frac{(b-a)^{n+2}\left[(-1)^{n+1}+1\right]}{2^{n+2}(n+2)} \mid \leq\left\{\begin{array}{l}
\frac{(b-a)^{n+2}}{2^{n+4}}(\Phi-\phi) \text { if } n \text { is even } \\
\frac{(b-a)^{n+2}}{2^{n+3}}(\Phi-\phi) \text { if } n \text { is odd }
\end{array}\right.
\end{aligned}
$$

and the inequality (3.10) is proved.

\section{References}

[1] A. M. Ostrowski, On an integral inequality, Aequat. Math., 4(1970), 358-373.

[2] P. Cerone, S. S. Dragomir, J. Roumeliotis and J. Sunde, A new generalistion of the trpezoid formula for n-time differentiable mappings and applications, Demonstratio Mathematica, 33(2000), 719-736.

[3] S. S. Dragomir and Th. M. Rassias (Ed.), Ostrowski Type Inequalities and Applications in Numerical Integration, Kluwer Academic Publishers, Dordrecht, 2002.

[4] S.S. Dragomir, Improvements of Ostrowski and generalised trapezoid inequality in terms of the upper and lower bounds of the first derivative, Tamkang J. Math., 34(2003), 213-222.

School of Comuter Science and Mathematics, Victoria University, PO Box 14428, MCMC 8001, Victoria, Australia.

E-mail: neil@csm.vu.edu.au

School of Comuter Science and Mathematics, Victoria University, PO Box 14428, MCMC 8001, Victoria, Australia.

E-mail: sever@csm.vu.edu.au 